\title{
Morteros de cementos alcalinos. Resistencia química al ataque por sulfatos y al agua de mar
}

\section{Alkaline cement mortars. Chemical resistance to sulfate and seawater attack}

\author{
F. PUERTAS(*), R. de GUTIERREZ(*) , A. FERNÁNDEZ-JIMÉNEZ(*), S. DELVASTO (*), J. MALDONADO (*) \\ (*) Instituto Eduardo Torroja (CSIC), Madrid, España \\ (*) Universidad del Valle, Cali, Colombia \\ Fecha de recepción: 13-III-02
}

Fecha de aceptación: 6-V-02

\section{RESUMEN}

Se ha estudiado la estabilidad química en medios sulfáticos y de agua de mar de morteros de escorias activadas alcalinamente y morteros de mezclas de escoria y cenizas volantes activadas alcalinamente. Se han empleado dos métodos para evaluar dicha estabilidad: Kock-Steinegger y la norma ASTM C1012. Se ha realizado una caracterización mineralógica y microestructural de los morteros (a diferentes edades de permanencia en los medios agresivos) a través de $D R X, S E M / E D X$ y porosimetria de mercurio.

Los resultados obtenidos han demostrado la elevada durabilidad de todos los morteros de cementos activados estudiados frente a la agresividad de los sulfatos y del agua de mar. Los morteros de escoria activada con $\mathrm{NaOH}$ son los más susceptibles al ataque por esos medios, con formación de productos expansivos como el yeso y la etringita, aunque en proporciones muy bajas.

\section{$S U M M A R Y$}

The durability and chemical resistance of alkali activated slag and fly ash/slag mortars in contact with sulfates and seawater media have been studied. Two methods were used in the evaluation of such durability: Kock-Steinegger and ASTM C1012. A mineralogical and a microstructural characterization of mortars were done at different ages of their conservation in aggressive media through XRD, SEM/ EDX and mercury porosimetry.

Results showed a high durability of activated cement mortars in sulfates and seawater media. $\mathrm{NaOH}$ activated mortars are the most sensitive to environment attack with formation of expansive products as gypsum and ettringite, although in very low proportion.
PALABRAS CLAVE: morteros de cementos alcalinos, cementos de escoria activada alcalinamente, durabilidad, ataque por sulfatos y agua de mar

\section{INTRODUCCIÓN}

Es conocida la mayor durabilidad en medios sulfáticos y de agua de mar, a medio y largo plazo, de hormigones que contienen escorias vítreas de horno alto (granuladas o peletizadas) como material cementante (1). Los factores que más influyen en dicha durabilidad son el régimen de curado y el tipo de cemento (contenido de $\mathrm{C}_{3} \mathrm{~A}$ y porcentaje de alúmina de la escoria).
KEYWORDS: alkaline cement mortars, alkali activated slag cements, durability, sulfate and sea water attack

\section{INTRODUCTION}

It is well known the high durability of concretes prepared with slag cements in sulfate and seawater environments (1). The most influencing factors of this durability are the curing methods, the type of cement $\left(C_{3} A\right.$ content and the alumina content in the slag). 
Estas escorias vítreas también pueden ser utilizadas en la preparación de cementos alternativos a los tradicionales cementos Portland. Estos cementos se obtienen por la activación alcalina de las escorias vítreas, y se caracterizan por presentar elevadas prestaciones mecánicas y obtenerse mediante un proceso más ecológico y con un menor consumo energético, que el requerido para la elaboración del clínker Portland. Este proceso está asociado a una elevada emisión de gases contaminantes a la atmósfera, a una alteración del paisaje por explotación de canteras y a un consumo energético debido a las elevadas temperaturas de clinkerización (superiores a los $1.450^{\circ} \mathrm{C}$ ) necesarias para completar las reacciones químicas.

Esos cementos de escorias activadas alcalinamente (AAS) fueron desarrollados a principio de los años 60 por Glukhovsky $(2,3)$. Desde entonces muchos investigadores han profundizado en su conocimiento, tanto desde el punto de vista de las reacciones y productos involucrados (4-7) como desde el punto de vista de su comportamiento físico y mecánico (8-12).

La durabilidad de estos cementos ha sido también abordada por diferentes autores (13-17). La microestructura y naturaleza de los productos de reacción en las pastas de escorias activadas difiere de la de una pasta de cemento Portland. La impermeabilidad de los morteros y hormigones de escorias activadas alcalinamente es superior a los elaborados con cementos Portland $(19,20)$. La velocidad de difusión de los iones $\mathrm{Cl}^{-}$en pastas de cementos de escorias activadas es de $30-40$ veces más lenta que en las pastas de cemento Portland, a igualdad de relaciones agua/ligante (16). Sus principales productos de reacción se caracterizan por una menor relación $\mathrm{C} / \mathrm{S}\left(\mathrm{CaO} / \mathrm{SiO}_{2}\right)$ y una menor solubilidad (18), lo que justificaría su mayor estabilidad química frente a diferentes agresivos externos. Los resultados obtenidos en torno a la estabilidad de morteros y hormigones de escorias activadas frente a medios agresivos de sulfatos y agua de mar, se refieren básicamente a los denominados cementos tipo F (16), y en general apuntan a una mayor resistencia química que los morteros y hormigones de cemento Portland. En el presente trabajo se ha estudiado la estabilidad química en medios sulfáticos y de agua de mar de morteros de escorias activadas que previamente habian sido optimizados desde un punto de vista mecánico (21). También se ha estudiado la estabilidad química en los mismos ambientes de morteros de cementos de mezclas de escorias y cenizas volantes activados alcalinamente.
Vitreous blast furnace slag can also be used to made alternative cements instead of the traditional ordinary Portland cement. Those cements are obtained from alkali-activation of vitreous slag, and they are characterized by high mechanical performances. They are obtained through an ecological and a low-cost energy process, contrary to Portland clinker process, which is associated to: a high rate of polluting gases -specially $\mathrm{CO}_{2}-$ to the atmosphere, alterations of the landscape due to the beneficiation of quarries, and high energy consume due to the high temperature (above $1.450^{\circ} \mathrm{C}$ ) necessary to reach the chemical reactions of clinkerization process.

During the early 60s, Glukhovsky et al developed cements manufactured with alkali-activated slag (AAS) $(2,3)$. Since then, a great deal of researchers have deepened into that knowledge, either from the viewpoint of the reactions and the products involved (4-7) or from the standpoint of the physical and the mechanical behaviour (8-12).

Different authors have approached the question of cement durability (13-17). Microstructure and nature of reaction products in pastes of activated slag differ from those of Portland cement paste. Alkali-activated slag mortars and concretes have a lower permeability than those made with Portland cement $(19,20)$. Diffusion rates of chloride ions (Cl) in pastes of activated slag cements are 30-40 times lesser than those reported by Portland cement pastes at equal water/cement ratio (16). The main reaction products from alkaline activation of slag are characterized by low $C / S$ $(\mathrm{CaO} / \mathrm{SiO})$ ratio and low solubility (18), which would justify their stronger chemical stability against different external agents. Results regarding chemical stability of mortars and concretes of activated slag in sulfates and sea water media are basically related to the so-called type $F$ cements (16), and generally imply stronger chemical resistance than that of mortars and concretes of Portland cement. This work is aimed to research the durability and chemical resistance in sulphate and seawater environments of activated slag mortars that previously have been optimized from a mechanical point of view (21). Also, chemical resistance of cement mortars made with the alkaline activation of a mixture of slag and fly ash has been studied in similar conditions. 


\section{EXPERIMENTAL}

\subsection{Materiales}

En la Tabla 1 se presenta el análisis químico y la superficie específica de la escoria vítrea de horno alto (Ensidesa, Avilés) y de la ceniza volante (procedente de la Central Térmica de Aboño) utilizadas. También se presenta el análisis químico y algunas propiedades físicas del cemento Portland utilizado en los ensayos comparativos sobre estabilidad de morteros en presencia de sulfatos.

El contenido en fase vítrea de la escoria de horno alto, determinado por DRX (22) y métodos microscópicos (23) es del orden del $95 \% \mathrm{El}$ análisis realizado por FTIR ha mostrado que mineralógicamente la escoria corresponde a una melilita con una composición próxima a $A_{5} G_{5}$ ( $A=$ akerminita, $G=$ gelenita). A través de DRX se ha confirmado que la ceniza volante es mayoritariamente un material amorfo que contiene cuarzo y mullita como fases cristalinas.

Para la activación alcalina de la escoria se han utilizado dos disoluciones activadoras: waterglass $\left(\mathrm{Na}_{2} \mathrm{SiO}_{3}+\right.$ $\mathrm{NaOH}+\mathrm{H}_{2} \mathrm{O}$ ) y $\mathrm{NaOH}$, ambas con la misma concentración de $4 \%$ en $\mathrm{Na}_{2} \mathrm{O}$ en masa de escoria (21).

En estudios previos (24) se comprobó que pastas con el $50 \%$ de escoria y $50 \%$ de cenizas volantes, activadas a temperatura ambiente con una disolución de $\mathrm{NaOH} 10 \mathrm{M}$, desarrollaban resistencias mecánicas a 28 días superiores a $50 \mathrm{MPa}$. Estas pastas fueron también seleccionadas para el estudio de estabilidad en medios sulfáticos.

\section{EXPERIMENTAL}

\subsection{Materials}

Table 1 shows the chemical composition and the specific surface of vitreous slag (blast furnace slag from Ensidesa, Avilés, Spain) and fly ash (from Aboño power plant, Spain). Moreover, chemical analysis and some physical properties of the Portland cement used as reference material are also presented in that Table.

Vitreous phase content of blast furnace slag was determined through XRD (22) and by microscopic methods (23), and it is about 95\%. Analysis through FTIR proved that slag, from a mineralogical standpoint, corresponds to a melilite with a composition close to $A_{5} G_{5}(A=$ akerminite, $G=$ gehlenite). Through XRD it was assessed that fly ash is mainly an amorphous material containing quartz and mullite as crystalline phases.

Focussing on alkaline activation of slag, two activating solutions were used: waterglass $\left(\mathrm{Na}_{2} \mathrm{SiO}_{3}\right.$ $\left.+\mathrm{NaOH}+\mathrm{H}_{2} \mathrm{O}\right)$ and $\mathrm{NaOH}$, both with the same concentration: $4 \% \mathrm{Na}_{2} \mathrm{O}$ in slag mass (21).

Previous studies (24) had proved that pastes with $50 \%$ slag and 50\% fly ash activated at room temperature with a solution of $\mathrm{NaOH} 10 \mathrm{M}$, after 28 days developed compression strengths higher than 50 MPa. These pastes were also chosen for the durability study in sulfate environments.

TABLA $1 / T A B L E I$

Composición química (\% en masa)

(Chemical composition (\% mass))

\begin{tabular}{|c|c|c|c|}
\hline & $\begin{array}{c}\text { CEM ENTO } \\
\text { Cement }\end{array}$ & $\begin{array}{c}\text { E SCOR IA } \\
\text { Slag }\end{array}$ & $\begin{array}{c}\text { CENIZA } \\
\text { VOLANTE } \\
\text { Fly ash }\end{array}$ \\
\hline $\mathrm{CaO}$ & 60.13 & 41.45 & 5.51 \\
\hline $\mathrm{SiO}_{2}$ & 20.75 & 35.50 & 51.49 \\
\hline $\mathrm{Al}_{2} \mathrm{O}_{3}$ & 6.53 & 12.15 & 29.03 \\
\hline $\mathrm{Fe}_{2} \mathrm{O}_{3}$ & 3.64 & 1.01 & 7.76 \\
\hline MgO & 2.56 & 8.34 & 2.35 \\
\hline $\mathrm{SO}_{3}$ & 1.97 & 0.18 & -- \\
\hline $\mathbf{S}^{2-}$ & & 0.92 & -- \\
\hline $\mathrm{Na}_{2} \mathrm{O}$ & & 0.58 & 0.66 \\
\hline $\mathrm{K}_{2} \mathrm{O}$ & & 0.64 & 2.83 \\
\hline TOTAL & $95.58+3,88$ (P.F.) & 100.77 & 99.63 \\
\hline Blaine $\left(\mathrm{m}^{2} / \mathrm{Kg}\right)$ & 350 & 460 & 380 \\
\hline $\begin{array}{l}\text { Resist. Compresión } \\
\text { (28dias) (MPa) } \\
\text { Compressive strength } \\
\text { (28 days) (Mpa) }\end{array}$ & 39.3 & & \\
\hline
\end{tabular}




\subsection{Ensayos y métodos}

La resistencia a los sulfatos y al agua de mar de morteros de escoria activada se evaluó utilizando los siguientes métodos: Kock-Steinegger (25) y la norma ASTM C1012 (26).

\subsubsection{Método de Kock-Steinegger}

Sobre las tres matrices: a) escoria activada con waterglass, $4 \%$ en $\mathrm{Na}_{2} \mathrm{O}$ en masa de escoria $\mathrm{b}$ ) escoria activada con $\mathrm{NaOH}, 4 \%$ en $\mathrm{Na}_{2} \mathrm{O}$ en masa de escoria, c) $50 \%$ escoria $+50 \%$ ceniza activada con $\mathrm{NaOH} 10 \mathrm{M}$; se aplicó el método de Kock-Steinegger. Mediante este método se evalúa la resistencia de morteros de cementos a la acción corrosiva de disoluciones acuosas sulfáticas. El comportamiento se estima a través de la evolución con el tiempo de la resistencia a flexotracción de los prismas de mortero sumergidos en las disoluciones agresivas.

Las probetas preparadas tenían las siguientes características:

- Un tamaño de $1 \times 1 \times 6 \mathrm{~cm}$. Se ensayaron 11 probetas por edad y medio

- Se utilizó una arena silícea con un 98\% de material cuarzoso y con un tamaño de grano comprendido entre 0,4 y $0,9 \mathrm{~mm}$

- Relación disolución activadora/escoria de 0,52

- Relación cemento/arena de 1/2

Los prismas fueron conservados inicialmente en cámara de humedad (>95\%) durante 21 dias, incluyendo las primeras 24 horas. A continuación los prismas fueron sumergidos totalmente en las siguientes disoluciones:

- Disolución acuosa de $\mathrm{Na}_{2} \mathrm{SO}_{4}$ al $4.4 \%$ en peso - Disolución de agua de mar artificial (ASTM D114 86)

- Agua desionizada/destilada, como medio de referencia

A las edades de 3, 7, 28, 90 y 180 días, los prismas fueron extraídos de las disoluciones y ensayados mecánicamente. Con el fin de conocer los procesos químicos involucrados, las transformaciones mineralógicas producidas en las probetas, y las alteraciones microestructurales de los morteros, a edades determinadas fueron estudiados por DRX, SEM/ EDX y porosimetría de mercurio.

\subsubsection{Método ASTM C 1012}

A partir de mezclas de escoria activada con waterglass $\left(\mathrm{Na}_{2} \mathrm{SiO}_{3}+\mathrm{NaOH}+\mathrm{H}_{2} \mathrm{O}\right)$ de concentración de $4 \%$ en $\mathrm{Na}_{2} \mathrm{O}$ en masa de escoria se prepararon probetas de mortero de relación arena:cementante de $2: 1$ y disolución activadora:escoria de 0,52 . Las probetas se

\subsection{Tests and methods}

The behaviour of activated mortars in sulfates and seawater media has been evaluated using the following methods: Kock-Steinegger (25), and ASTM C1012 standard (26).

\subsubsection{Kock-Steinegger method}

The Kock-Steinegger method was applied on three matrices : a) slag activated with waterglass, $4 \%$ of $\mathrm{Na}_{2} \mathrm{O}$ in slag mass; b) slag activated with $\mathrm{NaOH}, 4 \% \mathrm{Na}_{2} \mathrm{O}$ in slag mass; c) $50 \%$ slag $+50 \%$ fly ash activated with $\mathrm{NaOH}$ 10M. The Kock-Steinegger method evaluates the resistance of cement mortars to the corrosive action of aqueous sulfate solutions. The behavior of the material is estimated from the flexural strength of small mortar prisms submerged in a sodium sulfate solution. This strength is compared with that obtained from prisms stored in deionized water for the same period of time.

To apply the Kock-Steinegger method, mortar prisms of the following characteristics were prepared:

- Size of the prisms were $1 \times 1 \times 6 \mathrm{~cm}$. 11 test samples were tested each age and medium.

- Silicious sand was used (98\% wt $\mathrm{SiO}_{2}$ ) with a grain size between 0.4 and $0.9 \mathrm{~mm}$.

- Activator solution/slag ratio was 0.52 .

- Cement/sand ratio was $1: 2$.

Initially, prisms were kept in chamber at moisture $>95 \%$ for 21 days including first 24 hours. Following, prisms were immersed into the aggressive solutions:

- Aqueous $\mathrm{Na}_{2} \mathrm{SO}_{4}$ solution at $4.4 \%$ by weight.

- Artificial sea water solution (ASTM D114 86)

- Deionized/distilled water as a reference medium.

The prisms were removed from the solutions after 3, 7, 28, 90 and 180 days inmersion. At each age the flexural and compressive strengths were determined. In order to find out which chemical processes determine the durability and sulfate resistance, a mineralogical and a microstructural characterization of the mortars were conducted after storage. The mineralogical transformations were studied by $X R D$, and the microstructural study were performed by $S E M / E D X$ and mercury porosimetry.

\subsubsection{ASTM C 1012 method}

A mortar was prepared with a cement/sand ratio of $2: 1$ (using Ottawa type silica sand), and an activator solution/slag ratio of 0.52 . The alkaline solution used was waterglass $-\mathrm{Na}_{2} \mathrm{SiO}_{3}+\mathrm{NaOH}+\mathrm{H}_{2} \mathrm{O}$ - with a concentration of $4 \%$ of $\mathrm{Na}_{2} \mathrm{O}$ in slag mass. 
conservaron en cámara húmeda $(98 \% \mathrm{HR})$ por un período de 28 días y posteriormente se colocaron inmersas en una solución acuosa de Sulfato de Sodio $\left(\mathrm{Na}_{2} \mathrm{SO}_{4}\right)$ de concentración de $50 \mathrm{~g} / \mathrm{l}$ a pH controlado (6-8). A diferentes intervalos, hasta edad de 200 días, se registró la apariencia visual, el cambio dimensional y la resistencia a la compresión. Para el ensayo de resistencia mecánica se utilizaron probetas cúbicas de $50,8 \mathrm{~mm}$ de lado y arena silícea, tipo Ottawa. Como medio de comparación se utilizaron probetas preparadas en idénticas proporciones sumergidas en agua potable.

Los resultados obtenidos se compararon con los registrados sobre probetas de morteros preparadas con un cemento Portland sin adición, con relación arena:cemento de 2:1 y proporción de agua evaluada con base en el ensayo de fluidez, las cuales se sometieron a las mismas condiciones de inmersión en agua potable y disolución acuosa de $\mathrm{Na}_{2} \mathrm{SO}_{4}$

\section{RESULTADOS Y DISCUSIÓN}

\subsection{Método de Kock-Steinegger}

En las Tablas 2 y 3 se presentan los resultados de los ensayos mecánicos a flexotracción y compresión, respectivamente. El análisis de estas tablas muestra que las probetas de mortero de escoria activada con waterglass y las de mezcla de escoria/ceniza no experimentan variaciones significativas en sus valores resistentes cuando están sumergidas en las disoluciones agresivas, respecto a cuando están conservadas en el medio de referencia (agua desionizada/destilada); incluso se puede observar, en ocasiones, un incremento en las resistencias mecánicas. Únicamente, en las probetas de escoria activada con waterglass y conservadas en agua de mar se produce, a partir de los 90 días, una disminución en las resistencias a compresión, aunque esto no es observado en las mismas probetas ensayadas a flexotracción.

Sin embargo, las probetas de mortero de escorias activadas con $\mathrm{NaOH}$ manifiestan, en los dos medios agresivos, un descenso en sus resistencias mecánicas (ya sean a flexotracción como a compresión) que se puede estimar en torno al $15-25 \%$, respecto a las del medio de referencia.

El análisis mineralógico se ha realizado sobre porciones de las probetas ensayadas a 7 y 90 días, en todos los medios utilizados. En las Tablas 4, 5 y 6 se presentan los resultados de DRX, con una valoración de las fases
$50.8 \mathrm{~mm}$ side cubic prisms for mechanical strength tests were kept in moisture chamber $(98 \% H R)$ for 28 days. Afterwards, they were immersed into an aqueous solution of sodium sulfate $\left(\mathrm{Na}_{2} \mathrm{SO}_{4}\right)$ with a concentration of $50 \mathrm{~g} / \mathrm{l}$ and a controlled pH (6-8). At different intervals, until 200 days of storage of the prisms, the visual aspect, dimensional changes and compressive strengths were checked. In order to compare, test samples of the hardened mortar were immersed in tap water.

The results obtained were compared to those registered on mortar prisms prepared with Portland cement with no addition, with a sand:cement ratio of $2: 1$ and a water content evaluated on the basis of the fluidness test, which were submitted to the same soaking conditions in tap water and aqueous $\mathrm{Na}_{2} \mathrm{SO}_{4}$ solution.

\section{RESULTS AND DISCUSSION}

\subsection{Kock-Steinegger method}

Tables 2 and 3 show the results of flexural and compressive strength tests, respectively. From these Tables is deduced that waterglass activated slag cement mortars and those with a mixture of fly ash/slag do not have significant changes in their strength values when they are submerged in aggressive solutions, when compared to those maintained under reference conditions (deionized/distilled water). Only waterglass activated slag mortars immersed in seawater during 90 and 180 days show a minor increase rate of the compressive strengths, although this was not observed on the same samples tested to flexural strengths.

However, mortars prepared from $\mathrm{NaOH}$ activated slag immersed in sulfate aggressive solutions did not show increment of mechanical strengths (either to flexural and compression). This strength is around 15-25\% lower when compared to those conserved in deionized water.

Mineralogical analyses were made on portions from the samples tested at 7 and 90 days of storage in each media. XRD results are presented in Tables 4, 5 and 6. These mineralogical analyses were 
TABLA 2/TABLE 2

Valores de las resistencias a flexotracción

(Flexural strenght values)

\begin{tabular}{|c|c|c|c|}
\hline & AGUA/WATER & $\begin{array}{c}\text { AGUA DE MAR } \\
\text { SEAWATER }\end{array}$ & $\mathrm{Na}_{2} \mathrm{SO}_{4}$ \\
\hline $\begin{array}{l}\text { ESCORIA+WG } \\
S L A G+W G\end{array}$ & $\mathbf{R}_{\mathrm{F}}$ (MPa) & $\mathbf{R}_{\mathbf{F}}$ (MPa) & $\mathbf{R}_{\mathrm{F}}(\mathrm{MPa})$ \\
\hline 3días (days) & $2.4 \pm 0.3$ & $2.5 \pm 0.5$ & $2.6 \pm 0.5$ \\
\hline 7 días (days) & $4.1 \pm 0.6$ & $4.8 \pm 0.9$ & $3.2 \pm 0.4$ \\
\hline 28 días (days) & $6.0 \pm 0.5$ & $5.0 \pm 1.0$ & $5.2 \pm 0.7$ \\
\hline 90 días (days) & $9.6 \pm 0.6$ & $9.1 \pm 0.5$ & $10.3 \pm 1.3$ \\
\hline 180 días (days) & $10.9 \pm 1.5$ & $11.0 \pm 0.7$ & $10.9 \pm 0.9$ \\
\hline \multicolumn{4}{|l|}{$\begin{array}{c}\text { ESCORIA+ NaOH } \\
S L A G+\mathrm{NaOH} \\
\end{array}$} \\
\hline 3 días (days) & $4.0 \pm 0.2$ & $4.5 \pm 0.2$ & $4.5 \pm 0.5$ \\
\hline 7 días (days) & $3.8 \pm 0.2$ & $4.1 \pm 0.4$ & $4.1 \pm 0.5$ \\
\hline 28 días (days) & $3.7 \pm 0.3$ & $4.6 \pm 0.3$ & $4.7 \pm 0.4$ \\
\hline 90 días (days) & $5.5 \pm 0.3$ & $5.9 \pm 0.4$ & $4.3 \pm 0.4$ \\
\hline 180 días (days) & $6.4 \pm 0.7$ & $6.1 \pm 0.5$ & $4.7 \pm 0.4$ \\
\hline \multicolumn{4}{|l|}{$\begin{array}{c}\text { CENIZA/ESCORIA } \\
F L Y A S H / S L A G\end{array}$} \\
\hline 3 días (days) & $5.0 \pm 0.2$ & $5.2 \pm 0.2$ & $5.1 \pm 0.1$ \\
\hline 7 días (days) & $5.0 \pm 0.2$ & $5.1 \pm 0.2$ & $5.1 \pm 0.4$ \\
\hline 28 días (days) & $6.4 \pm 0.2$ & $6.1 \pm 0.2$ & $6.5 \pm 0.2$ \\
\hline 90 días (days) & $7.7 \pm 0.3$ & $7.8 \pm 0.4$ & $7.4 \pm 0.2$ \\
\hline 180 días (days) & $7.6 \pm 0.4$ & $\cdot 7.4 \pm 0.1$ & $7.7 \pm 0.3$ \\
\hline
\end{tabular}

TABLA $3 / T A B L E 3$

Valores de resistencia a compresión

(Compressive strength values)

\begin{tabular}{|c|c|c|c|}
\hline & AGUA/WATER & $\begin{array}{c}\text { AGUA DE MAR } \\
S E A W A T E R\end{array}$ & $\mathrm{Na}_{2} \mathrm{SO}_{4}$ \\
\hline $\begin{array}{c}\text { ESCORIA +W G } \\
S L A G+W G\end{array}$ & $R_{C}(M p a)$ & $\mathrm{R}_{\mathbf{C}}(\mathbf{M P a})$ & $\mathbf{R}_{\mathbf{C}}(\mathbf{M P a})$ \\
\hline 3días (days) & $11.9 \pm 0.7$ & $14.1 \pm 1.9$ & $12.8 \pm 1.7$ \\
\hline 7 días (days) & $32.5 \pm 2.4$ & $33.1 \pm 2.2$ & $23.5 \pm 8.1$ \\
\hline 28 días (days) & $34.0 \pm 2.9$ & $32.2 \pm 3.0$ & $36.0 \pm 3.2$ \\
\hline 90 días (days) & $55.4 \pm 2.0$ & $47.1 \pm 3.1$ & $84.3 \pm 2.3$ \\
\hline 180 días (days) & $81.2 \pm 1.3$ & $59.2 \pm 1.7$ & $100.9 \pm 2.4$ \\
\hline \multicolumn{4}{|l|}{$\begin{array}{c}\text { ESCORIA }+\mathrm{NaOH} \\
S L A G+N a O H\end{array}$} \\
\hline 3 días (days) & $33.6 \pm 0.6$ & $35.1 \pm 1.2$ & $35.5 \pm 1.2$ \\
\hline 7 días (days) & $28.9 \pm 1.4$ & $29.3 \pm 0.9$ & $29.5 \pm 2.0$ \\
\hline 28 días (days) & $31.8 \pm 2.0$ & $32.7 \pm 1.9$ & $36.1 \pm 1.9$ \\
\hline 90 días (days) & $42.9 \pm 1.5$ & $42.4 \pm 2.4$ & $37.7 \pm 1.9$ \\
\hline 180 días (days) & $43.2 \pm 2.9$ & $36.4 \pm 2.8$ & $33.3 \pm 2.2$ \\
\hline \multicolumn{4}{|c|}{$\begin{array}{c}\text { CENIZA/ESCORIA } \\
F L Y A S H / S L A G \\
\end{array}$} \\
\hline 3 días (days) & $22.5 \pm 0.4$ & $21.5 \pm 1.2$ & $22.2 \pm 0.4$ \\
\hline 7 días (days) & $23.6 \pm 0.7$ & $23.4 \pm 1.4$ & $23.4 \pm 0.7$ \\
\hline 28 días (days) & $30.7 \pm 0.7$ & $24.7 \pm 2.7$ & $31.6 \pm 0.6$ \\
\hline 90 días (days) & $37.2 \pm 0.8$ & $29.7 \pm 5.8$ & $37.8 \pm 1.2$ \\
\hline 180 días (days) & $38.2 \pm 1.2$ & $35.9 \pm 1.0$ & $37.0 \pm 1.4$ \\
\hline
\end{tabular}




\section{TABLA 4/TABLE 4}

Resultados de DRX. Morteros de escoria activada con waterglass (XRD results. Waterglass activated slag cement mortars)

\begin{tabular}{|c|c|c|c|c|c|c|c|c|c|}
\hline & $\mathbf{Q}$ & $\mathbf{M}$ & $\mathrm{C}$ & $\mathrm{V}$ & A & H & $\mathrm{C}-\mathrm{S}-\mathrm{H}$ & $\mathbf{Y}$ & $\mathbf{E}$ \\
\hline $\begin{array}{l}\text { A gua } \\
\text { water } \\
7 \text { dias } \\
7 \text { days }\end{array}$ & ++ & -- & ++ & o & -- & -- & o & -- & -- \\
\hline $\begin{array}{c}\text { A gua } \\
\text { water } \\
90 \text { dias } \\
90 \text { days }\end{array}$ & +++ & -- & ++ & + & -- & -- & o & -- & -- \\
\hline $\begin{array}{c}\text { M ar } \\
\text { Sea } \\
7 \text { días } \\
7 \text { days }\end{array}$ & +++ & -- & ++ & $\mathbf{0}$ & -- & -- & 0 & -- & -- \\
\hline $\begin{array}{c}\text { M ar } \\
\text { Sea } \\
90 \text { días } \\
90 \text { days }\end{array}$ & +++ & -- & ++ & + & -- & -- & $\mathbf{0}$ & + & -- \\
\hline $\begin{array}{c}\mathrm{Na}_{2} \mathrm{SO}_{4} \\
7 \text { días } \\
7 \text { days }\end{array}$ & +++ & -- & ++ & о & -. & -- & 0 & -- & -- \\
\hline $\begin{array}{l}\mathrm{Na}_{2} \mathrm{SO}_{4} \\
90 \mathrm{días} \\
90 \text { days }\end{array}$ & +++ & -. & ++ & o & -- & -. & 0 & -- & -- \\
\hline
\end{tabular}

$\mathrm{Q}=\mathrm{Cu}$ arzo, $\mathrm{M}=\mathrm{M}$ ullita, $\mathrm{C}=\mathrm{C}$ alcita, $\mathrm{V}=\mathrm{V}$ aterita, $\mathrm{A}=\mathrm{A}$ ragonito, $\mathrm{C}-\mathrm{S}-\mathrm{H}=\mathrm{gcl} \mathrm{C}-\mathrm{S}-\mathrm{H}, \mathrm{Y}=\mathrm{Y}$ eso, $\mathrm{E}=\mathrm{E}$ tringita, $\mathrm{H}=\mathrm{H}$ id rotalc ita

$+++=$ abundante,$++=$ moderado, $+=$ poco, $0=\operatorname{trazas},-.=$ ausente

$Q=Q$ uartz, $\quad M=$ Mullite,$\quad C=$ Calcite,$\quad V=$ Vaterite, $\quad A=$ Aragonite, $\quad C-S-H=g e l \quad C-S-H$, $Y=G$ ypsyum, E E tringite, $H=H$ idrotalcite

$+++=a b u n d a n t,++=$ moderate, $+=$ litte, $o=$ traces,,$-=a b s e n t$

TABLA 5/TABLE 5

Resultados de DRX. Morteros de escoria activada con $\mathrm{NaOH} /(X R D$ results. NaOH activated slag cement mortars)

\begin{tabular}{|c|c|c|c|c|c|c|c|c|c|}
\hline & $\mathbf{Q}$ & $\mathbf{M}$ & $\mathrm{C}$ & $\mathrm{V}$ & $\mathbf{A}$ & $\mathbf{H}$ & $\mathrm{C}-\mathrm{S}-\mathrm{H}$ & $\mathbf{Y}$ & $\mathbf{E}$ \\
\hline $\begin{array}{l}\text { A gua } \\
\text { water } \\
7 \mathrm{dias} \\
7 \text { days }\end{array}$ & ++ & -- & ++ & -- & -- & + & 0 & -- & -- \\
\hline $\begin{array}{c}\text { A gua } \\
w \text { ater } \\
90 \text { dias } \\
90 \text { days }\end{array}$ & +++ & -- & ++ & 0 & + & + & + & -- & -- \\
\hline $\begin{array}{c}\text { M ar } \\
\text { Sea } \\
7 \text { dias } \\
7 \text { days }\end{array}$ & +++ & -- & ++ & - & - & + & + & -- & $\cdots$ \\
\hline $\begin{array}{c}\text { M ar } \\
\text { Sea } \\
90 \text { dias } \\
90 \text { days }\end{array}$ & +++ & -- & $+t$ & $-\infty$ & + & + & o & + & -- \\
\hline $\begin{array}{c}\mathrm{Na}_{2} \mathrm{SO}_{4} \\
7 \mathrm{dias} \\
7 d a y s\end{array}$ & +++ & -- & ++ & $\cdots$ & $\ldots$ & + & + & -- & $\cdots$ \\
\hline $\begin{array}{l}\mathrm{N}_{2} \mathrm{SO}_{4} \\
90 \mathrm{dias} \\
90 d a y s\end{array}$ & +++ & - & $+t$ & -- & 0 & + & 0 & -- & 1 \\
\hline
\end{tabular}

$\mathrm{Q}=\mathrm{Cuarzo}, \mathrm{M}=\mathrm{M}$ ullita, $\mathrm{C}=\mathrm{C}$ alcita, $\mathrm{V}=\mathrm{V}$ aterita, $\mathrm{A}=\mathrm{A}$ ragonito, C $-\mathrm{S}-\mathrm{H}=\mathrm{gel} \mathrm{C}-\mathrm{S}-\mathrm{H}, \mathrm{Y}=\mathrm{Y}$ eso, $\mathrm{E}=\mathrm{E}$ tring ita, $\mathrm{H}=\mathrm{H}$ idrotalcita

$+++=$ abundante, $++=$ moderado, $+=$ poco, $0=\operatorname{trazas},-=$ ausente

$Q=Q$ uartz, $\quad M=\quad M$ ullite, $C=C$ alcite, $\quad V=V$ aterite, $A=A$ ragonite, C-S-H=gel C-S-H, $Y=G$ ypsy $\mathrm{m}, E=E$ tringite, $H=H$ idrotalcite

$+++=a b u n d a n t,++=m o d e r a t e,+=$ litte, $,+=\operatorname{traces},--=a b \operatorname{sent}$ 
Resultados de DRX. Morteros de mezclas de ceniza volante y escoria activados (XRD results. Activated fly ash/slag mix cement mortars)

\begin{tabular}{|c|c|c|c|c|c|c|c|c|c|}
\hline & $\mathbf{Q}$ & $\mathbf{M}$ & $\mathrm{C}$ & $\mathrm{V}$ & $\mathbf{A}$ & $\mathbf{H}$ & $\mathrm{C}-\mathrm{S}-\mathrm{H}$ & $\mathbf{Y}$ & $\mathbf{E}$ \\
\hline $\begin{array}{l}\text { A gua } \\
\text { Water } \\
7 \text { días } \\
7 \text { days }\end{array}$ & +++ & + & ++ & -- & -- & + & o & -- & -- \\
\hline $\begin{array}{c}\text { Agua } \\
\text { water } \\
90 \text { días } \\
90 \text { days }\end{array}$ & +++ & + & ++ & -- & -- & + & o & -- & -- \\
\hline $\begin{array}{c}\text { Mar } \\
\text { Sea } \\
7 \text { días } \\
7 \text { days }\end{array}$ & +++ & + & ++ & -- & -- & + & $\mathbf{0}$ & -- & -- \\
\hline $\begin{array}{c}\text { Mar } \\
\text { Sea } \\
90 \text { días } \\
90 \text { days }\end{array}$ & +++ & + & ++ & -- & -- & + & $?$ & -- & -- \\
\hline $\begin{array}{c}\mathrm{Na}_{2} \mathrm{SO}_{4} \\
7 \text { dias } \\
7 \text { days }\end{array}$ & +++ & + & ++ & -- & -. & + & $\mathbf{0}$ & -- & -- \\
\hline $\begin{array}{l}\mathrm{Na}_{2} \mathrm{SO}_{4} \\
90 \text { días } \\
90 \text { days }\end{array}$ & +++ & + & ++ & -- & -- & + & $\mathbf{0}$ & -- & -- \\
\hline
\end{tabular}

$\mathrm{Q}=\mathrm{C}$ uarzo, $\mathrm{M}=\mathrm{M}$ ullita, $\mathrm{C}=\mathrm{C}$ alcita, $\mathrm{V}=\mathrm{V}$ aterita, A = A ragonito, C $-\mathrm{S}-\mathrm{H}=\mathrm{gel} \mathrm{C}-\mathrm{S}-\mathrm{H}, \mathrm{Y}=\mathrm{Y}$ eso, $\mathrm{E}=\mathrm{E}$ tringita, $\mathrm{H}=\mathrm{H}$ idrotalcita

$+++=$ abundante,$++=$ moderado, $+=$ poco, $o=\operatorname{trazas},--=$ ausente

$Q=Q$ uartz,$\quad M=$ Mullite,$\quad C=$ Calcite,$\quad V=$ Vaterite,$\quad A=$ Aragonite, $\quad C-S-H=g e l \quad C-S-H$,

$Y=G$ ypsyum, E= Etringite, $H=$ Hidrotalcite

$+++=$ abundant, $++=$ moderate, $+=$ litte, $o=$ traces, $--=a b \operatorname{sen} t$

cristalinas identificadas. Este análisis mineralógico se ha realizado sobre muestras enriquecidas en pastas, para lo cual se intentó reducir en lo posible el contenido en arena cuarzosa del árido. Sin embargo, a pesar de ello, en todas las muestras había una cantidad muy importante de cuarzo, siendo el componente cristalino mayoritario en todas las muestras.

El principal producto de reacción en las pastas de escoria activada alcalinamente es un silicato cálcico hidratado (similar al gel C-S-H presente en una pasta de cemento Portland, aunque con algunas diferencias estructurales y composicionales). Los resultados obtenidos han mostrado que la ordenación estructural (cristalinidad) de ese silicato cálcico hidratado está muy relacionada con la naturaleza del activador. Cuando el activador es $\mathrm{NaOH}$, las líneas de difracción características de ese compuesto (12.59 $\AA$ y $3.07 \AA$ ) se observan con mayor claridad que en las pastas activadas con waterglass, indicando que el silicato cálcico hidratado formado cuando el activador es $\mathrm{NaOH}$, tiene un mayor orden estructural o mejor cristalización. En estas pastas también se identifica la fase hidrotalcita $\left(\mathrm{Mg}_{6} \mathrm{Al}_{2} \mathrm{CO}_{3}(\mathrm{OH})_{16} \cdot 4 \mathrm{H}_{2} \mathrm{O}\right)$, que no se observa en las pastas activadas con waterglass. Junto a estos productos de reacción, en estas pastas también se carried out on samples enriched in pastes; The content of quartz sand of the aggregate was reduced as much as possible. Though, in spite of this fact, there was a considerable amount of quartz in all samples, being the main crystalline phase in all samples.

The main reaction product in alkali-activated slag pastes is a hydrate calcium silicate (similar to the $C-S$-H gel present in Portland cement pastes, although with some structural and compositional differences). Results proved that the structural order of this hydrate calcium silicate is closely related to the nature of the alkaline activator used. When activator was $\mathrm{NaOH}$, characteristic diffraction lines due to this compound $(12.59 \AA, 3.07 \AA)$ were observed. These reflections were not so clearly detected in the diffractogramms of waterglass activated samples. From these results is deduced that the hydrate calcium silicate formed when the activator is $\mathrm{NaOH}$ has a major structural order or better crystallization than that formed when the activator is waterglass. In the $\mathrm{NaOH}$ activated matrices the phase called hydrotalcite $\left.\left(\mathrm{Mg}_{6} \mathrm{Al}_{2} \mathrm{CO}_{3} \mathrm{OH}\right)_{16} \cdot 4 \mathrm{H}_{2} \mathrm{O}\right)$ was also identified, but this phase was not observed in waterglass activated samples. In these pastes, besides reaction products, some carbonated phases were identified. In all samples the presence of calcium 
identifican unas fases carbonatadas. En todas las muestras hay presencia de carbonato cálcico (calcita). En las pastas de escorias activadas con waterglass se identifica también vaterita (no se descarta que haya algo de aragonito, pero en muy baja proporción); mientras que en las pastas activadas con $\mathrm{NaOH}$ junto a la calcita se encuentra preferentemente aragonito. La presencia de estos carbonatos se explica fundamentalmente por la carbonatación de las muestras durante el período de curado (21 días), previo a sumergirlas en las disoluciones agresivas. Esos carbonatos son observados ya desde el tercer día de conservación de los prismas.

En las muestras sumergidas en las disoluciones de agua de mar se identifica a los 90 días de conservación, yeso; mientras que en las mantenidas en la disolución de $\mathrm{Na}_{2} \mathrm{SO}_{4}$, únicamente se observa en el mortero de escoria activada con $\mathrm{NaOH}$, etringita a nivel de trazas.

En las muestras de escoria y ceniza volante activadas alcalinamente se identifica por DRX también un silicato cálcico hidratado como producto de reacción junto a la hidrotalcita. En las muestras conservadas en los medios agresivos no se identifica ningún compuesto resultante de la interacción química. No se detecta la presencia de yeso ni de etringita.

En la Figura 1 se presenta la porosidad total de las probetas a 7 y 90 días, obtenidas mediante porosimetría de mercurio, en todas las muestras carbonate (calcite) was detected. Other form of calcium carbonate as vaterite was also found in slag pastes activated with waterglass (the possible existence of some aragonite is not rejected, but in a very low proportion). In pastes activated with $\mathrm{NaOH}$ together with calcite, aragonite was mainly found. The presence of these carbonates can be explained by the carbonation of the mortars during the initial curing period (21 days), previously to immersion into aggressive solutions. Those carbonates were already observed after the third day of the prisms inmersion process.

After 90 days of storage, gypsum was identified in samples submerged into sea-water solution. Only traces of ettringite were detected (at traces level) in mortars of slag activated with $\mathrm{NaOH}$ immersed in $\mathrm{Na}_{2} \mathrm{SO}_{4}$

In samples prepared from the alkaline activation of fly ash/slag mixtures, a hydrate calcium silicate was identified as reaction product together with hydrotalcite. In samples preserved in aggressive solutions, no compounds resulting from a chemical interaction were found. No presence of gypsum or ettringite were detected either.

Figure 1 shows the total porosity of prisms at 7 and 90 days, obtained through mercury porosimetry. This

Figure points out that the waterglass activated slag

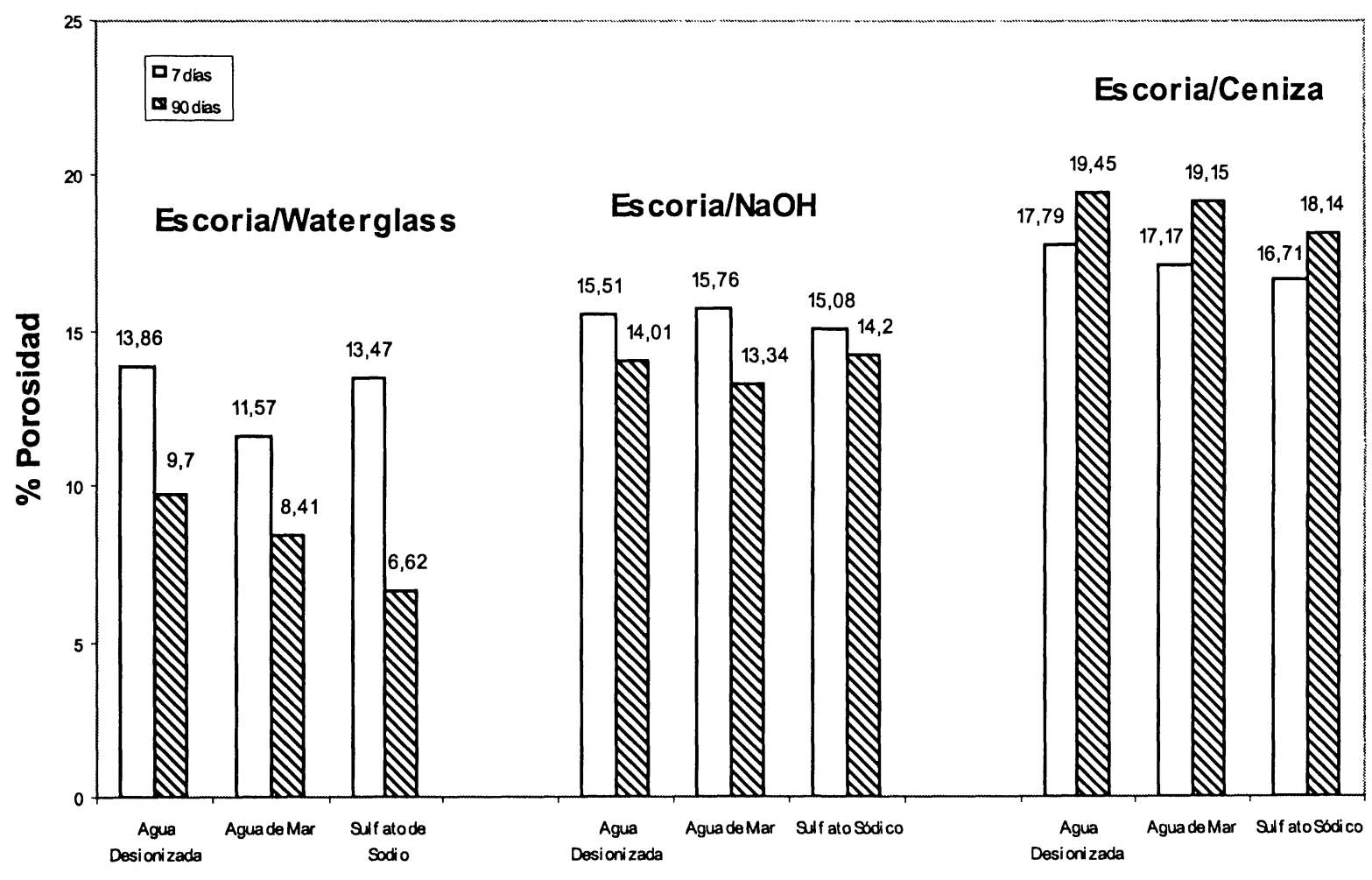

Figura 1.- Evolución de la porosidad total en los distintos morteros y medios agresivos.

Figure 1.- Evolution of total porosity in the diffferent mortars and aggressive media. 
estudiadas y en los tres medios de ensayo. De esta figura se desprende que las porosidades más bajas se obtienen en los morteros de escorias activadas con waterglass, con un descenso muy marcado en la porosidad total desde los 7 a los 90 días de inmersión (oscila entre el 30 al 50\%). Los morteros sumergidos en las disoluciones agresivas experimentan a los 90 días reducciones en la porosidad total superiores a los producidos en los morteros conservados en agua desionizada/destilada. Los morteros de escoria activada con $\mathrm{NaOH}$ tienen valores de porosidad total superiores a los de escoria activada con waterglass, y los descensos producidos con el tiempo de inmersión son menores (alrededor de un 10\%). El medio agresivo no parece influir en la porosidad total de estos morteros, ya que los valores obtenidos son similares en los tres casos.

Finalmente, las porosidades totales de los morteros de escoria y ceniza activadas alcalinamente son muy superiores a las evidenciadas por los otros morteros y además evolucionan de manera diferente. En los tres medios estudiados se observa un incremento en la porosidad total con el tiempo de inmersión, siendo mayores las porosidades a los 90 días que a los 7 días de inmersión. Los medios agresivos tampoco parecen influir en los valores de porosidad total.

En la Figura 2 se muestra la distribución del tamaño de los poros respecto a la porosidad total de cada mortero en todos los medios estudiados. Aunque es discutida la utilización de la porosimetría de mercurio como técnica adecuada para determinar la distribución de tamaños de poro en morteros y hormigones (27), en nuestro caso es empleada a modo comparativo, dada la igualdad de condiciones de obtención. Los resultados demuestran que en los morteros de escorias activadas con waterglass a los 7 días de conservación en todos los medios de estudio, los poros de tamaño comprendido entre 1 y $0,1 \mu \mathrm{m}$ representaban más del $50 \%$ de los poros; a los 90 días alrededor del $80 \%$ de los poros tienen tamaños entre 0,1 y $0,01 \mu \mathrm{m}$. Es decir, se produce una reducción muy importante de la porosidad total y una disminución en el tamaño de los poros. Este efecto se observa en todos los medios de conservación, ya sea agua destilada o soluciones sulfáticas. Ese refinamiento en el tamaño de los poros de estos morteros es el resultado de la deposición de productos de reacción y ubicación espacial de estos, procedentes de la activación alcalina de la escoria, los productos expansivos (formados en muy pequeña cantidad) no parecen afectar al sistema poroso de estos morteros. Como resultado de tal refinamiento los morteros son más impermeables y resistentes al ataque químico externo.

En los morteros de escoria activada con $\mathrm{NaOH}$ no se observan modificaciones en el tamaño de los poros, ni mortar samples have the lower porosity and a very remarkable decrease of total porosity from 7 to 90 immersion days (it oscillates between 30 and $50 \%$ ). After 90 days, mortars immersed in aggressive solutions undergo reductions in total porosity higher than those conserved in deionized/distilled water. Slag mortars activated with $\mathrm{NaOH}$ have total porosity values higher than those activated with waterglass, and the decrease of porosity developed during immersion time are lesser than 10\%. Sulfate aggressive solutions do not seem to influence the total porosity.

Finally, total porosity of hardened fly ash/slag activated mortars is much higher than that of the other activated mortars. After the immersion in sulfate aggressive solution an increase of the total porosity is observed in these mortars.

Figure 2 shows pore size distribution as compared to total porosity of each mortar in all studied media. Although mercury porosimetry does not seem to be the more adequate technique to determine pore size distribution in mortars and concretes (27), in our case it has been used as a comparative tool in equal conditions. Experimental results proved that more than $50 \%$ of the pores in waterglass activated slag mortar prisms after 7 days in a sulphate solution and in deionized water, had sizes between 1 and $0.1 \mu \mathrm{m}$. At 90 curing days, when around $80 \%$ of the pores reported sizes between 0.1 and $0.01 \mu \mathrm{m}$. This means, that they underwent a very important reduction of the total porosity and a decrease of the pore size. This pore size refinement could be explained as the result of the precipitation of new reaction products, generated by the alkali-activation of the slag. This effect is observed both in mortars preserved in deionized water and in sulfate solutions. The expansion products formed in very little amount did not seem to affect the porous system of these hardened mortars. This refinement must yield mortars with less permeability and more chemical resistance against external chemical attacks.

In slag mortars activated with $\mathrm{NaOH}$ no changes were observed in pore size distribution, either due to 
por efecto del tiempo de reacción y conservación (únicamente una ligera disminución en la porosidad total) ni por el medio de conservación de las probetas. En todos los morteros estudiados, el $40-50 \%$ de los poros tiene un tamaño comprendido entre $0,1-0,01 \mu \mathrm{m}$, el resto de los poros se encuentran entre 10-1 $\mu \mathrm{m}$ $(\sim 30 \%)$ y $1-0,1 \mu \mathrm{m}(\sim 20 \%)$. La permanencia de las probetas en los medios agresivos no afecta a la distribución del tamaño de poros. Una diferencia the effect of reaction time or by conditions of storage but only a slight decrease of the total porosity. In all mortars studied, between 40 to $50 \%$ of the pores had a size between 0.1 to $0.01 \mu \mathrm{m}$, the rest of the pores were between 10 and $1 \mu \mathrm{m}(\sim 30 \%)$ and between 1.0- $0.10 \mu \mathrm{m}$ ( 20\%). The inmersion age of mortar samples in sulphate solutions did not affect the pore size distribution. The remarkable difference between hardened activated slag mortars with $\mathrm{NaOH}$ and those

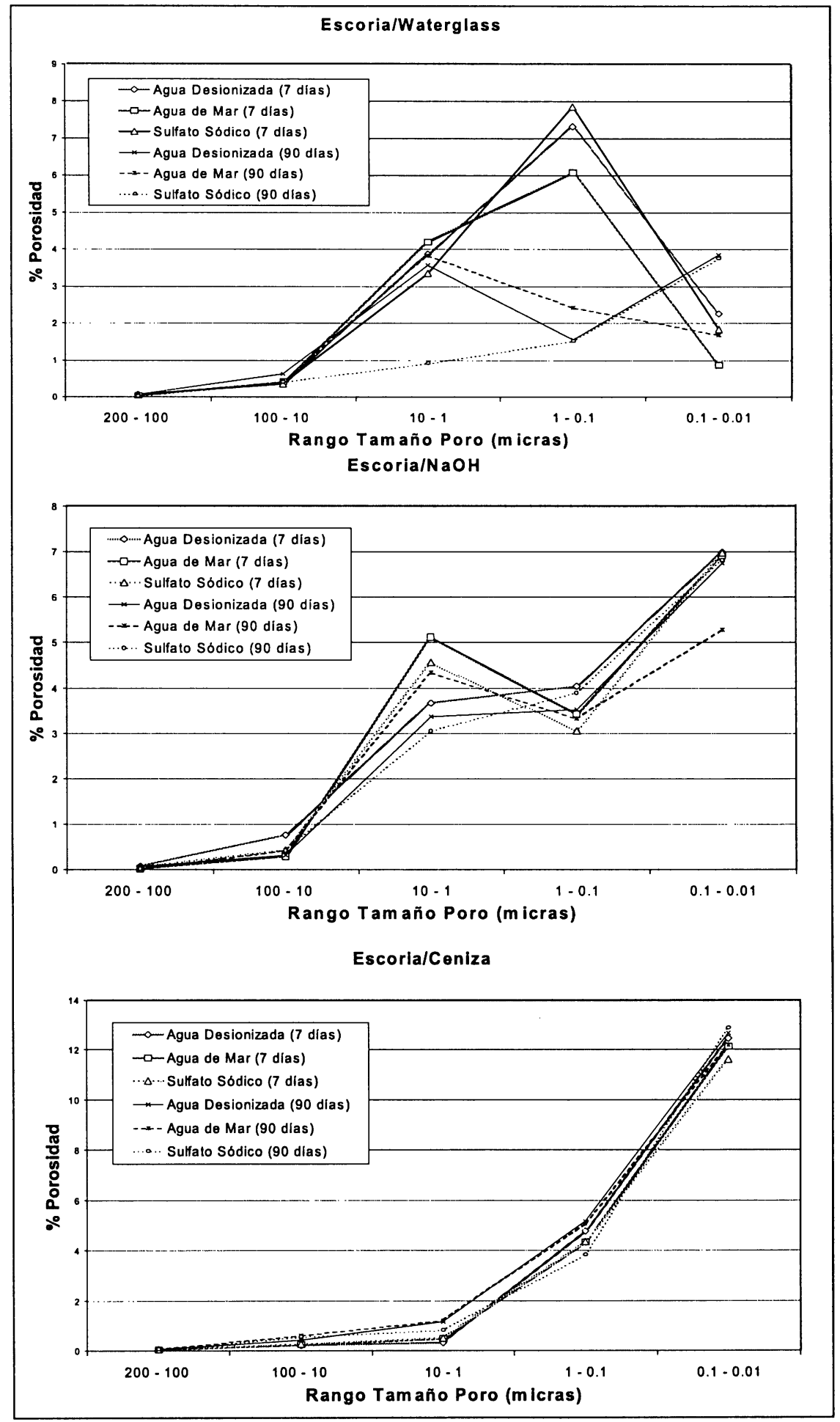

Figura 2.- Distribución de tamaño de poros en los morteros.

Figure 2.- Pore size distribution in mortars. 
significativa entre los morteros de escoria activada con $\mathrm{NaOH}$ y los activados con waterglass es la mayor proporción, en los primeros, de poros más pequeños $(0,1-0,01 \mu \mathrm{m})$. La porosidad total de esos morteros es mayor aunque el tamaño de sus poros es menor. Este fenómeno se ha asociado a la cinética del proceso de activación y a las diferencias estructurales y composicionales del principal producto de reacción (21).

Los morteros de escoria y ceniza volantes activados alcalinamente tienen porosidades totales mayores que los anteriores morteros, sin embargo, el tamaño de sus poros es sensiblemente inferior. Más del $70 \%$ de los poros de esos morteros son inferiores a $0,1-0,01 \mu \mathrm{m}$; es decir, son morteros con elevada porosidad pero con poros de muy pequeño tamaño. Los morteros conservados en los medios sulfáticos manifiestan una distribución de tamaño de poros prácticamente idéntica a la presentada en el medio de referencia (agua desionizada). La presencia de ceniza volante en estos morteros y su activación puede explicar este comportamiento de la porosidad en estas pastas. Las cenizas volantes que no reaccionan actúan como un inerte rellenando poros y compactando el material; por otra parte, y como resultado de la activación alcalina de las cenizas volantes, se forma un polímero inorgánico de naturaleza amorfa y cohesiva, que contribuye a la reducción de los poros grandes e incrementa las resistencias. Todo ello contribuye a generar unos materiales de elevada impermeabilidad y gran resistencia química, explicando la elevada estabilidad de estos morteros en los medios agresivos estudiados.

El estudio microestructural realizado por SEM/EDX ha confirmado la formación de algunos productos resultantes de la interacción entre los morteros de escoria activada con $\mathrm{NaOH}$ y las disoluciones de agua de mar y $\mathrm{Na}_{2} \mathrm{SO}_{4}$. Se ha observado la presencia, en poros de gran tamaño, de unas partículas aglomeradas, que al ser observadas a mayores aumentos, son cristales en forma de agujas. El análisis (realizado por EDX) sobre dichos cristales muestra que los elementos presentes son: $\mathrm{Ca}, \mathrm{S}$ y Al. Las Figuras 3 y 4 se presentan unas micrografias que muestran lo anteriormente descrito. A través de DRX se identificó yeso, en los morteros conservados en agua de mar, y ettringita en los mantenidos en disolución de sulfato sódico. A través de $\mathrm{SEM} / \mathrm{EDX}$ no se confirmó la presencia de yeso, aunque si se identificó ettringita.

En los morteros de escoria activada con waterglass conservados en las disoluciones agresivas no se encontró ningún producto que contuviera azufre en su composición. En los morteros de escoria y ceniza activados alcalinamente no se identificó ningún producto resultante de la interacción del mortero y las disoluciones agresivas. activated with waterglass is that in the former there was a higher proportion of smaller pores (0,1-0.01 $\mu \mathrm{m})$. Total porosity of those $\mathrm{NaOH}$ activated slag mortars were higher although pore size were smaller. This phenomenon is associated to kinetics of the activation process and to structural and compositional differences of the main reaction product (21).

Alkali activated fly ash/slag mortars had a total porosity higher than the above-mentioned mortars, in spite of this fact their pore size was noteworthy smaller. More than $70 \%$ of the pores of those mortars had a range of size smaller than 0.1-0.01 $\mu \mathrm{m}$, which means they were high porosity mortars but pores were very small. Hardened mortars maintained in sulfate solutions showed a pore size distribution practically identical to those specimens immersed in the reference medium (deionized water). Existence of fly ash particles in those mortars and their activation can explain this behavior. Fly ash particles unreactive is can act as inert, clogging pores and compacting the material; on the other hand, as a result of alkaliactivation of fly ash, an amorphous and cohesive inorganic polymer is formed. Both cause a reduction of large pores and a increase of mechanical resistances. This process contributes to create materials of low permeability and high chemical strength, which explains the strong stability of these mortars in aggressive sulfate solutions.

Microstructural study through SEM/EDX evidences the presence of products formed as result of the interaction between $\mathrm{NaOH}$ activated mortars and sea water and $\mathrm{Na}_{2} \mathrm{SO}_{4}$ solutions. In large size pores of these mortars, the presence of needle-shaped crystals agglomerated particles was detected. Their analysis (through EDX) showed $\mathrm{Ca}, \mathrm{S}$ and Al. Figures 3 and 4 illustrate what has been described. Through XRD, gypsum was detected in activated slag mortars conserved in seawater, and also traces of ettringite in those storaged in sodium sulfate solution. Through SEM/EDX no presence of gypsum could be proved; although, some ettringite was found.

In waterglass activated slag mortars inmersed in aggressive solutions, no products containing $S$ were found. In mortars made of alkali-activated fly ash/slag mixtures was no identified any product resulting from interaction of mortar and aggressive solutions. 


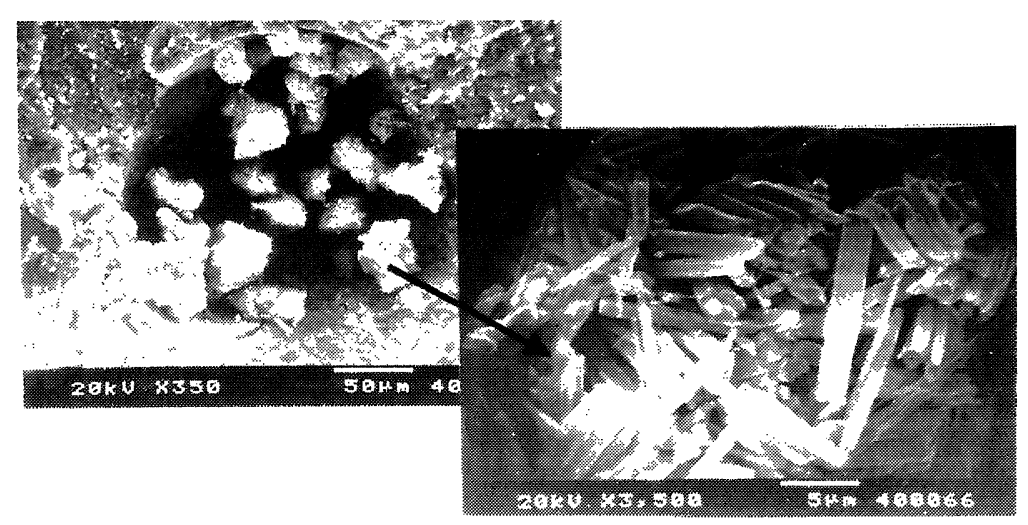

Figure 3.- Morteros de escoria activada con $\mathrm{NaOH}$ conservada en agua de mar (90 días). Poros con partículas aglomeradas. Detalle de las partículas. con forma de aguja.

Figure 3.- $\mathrm{NaOH}$ activated slag mortars conserved in seawater (90 days). Pores with agglomerated particles. Detail of needle-shaped particles.

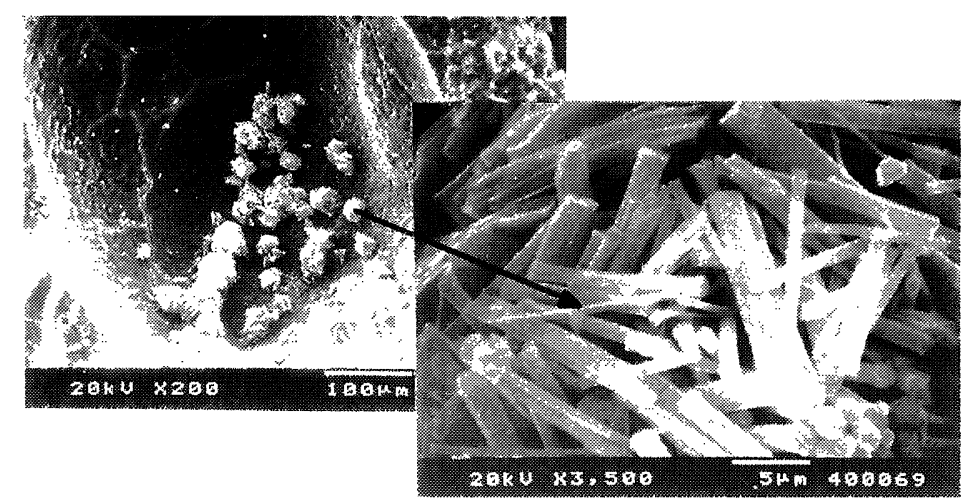

Figure 4.- Mortero de escoria activada con $\mathrm{NaOH}$ conservada en $\mathrm{Na}_{2} \mathrm{SO}_{4}(90$ días). Poros con partículas aglomeradas. Detalle de las partículas

Figure 4.- $\mathrm{NaOH}$ activated slag mortars conserved in $\mathrm{Na}_{2} \mathrm{SO}_{4}(90$ days). Pores with agglomerated particles. Detail of particles.

De los resultados presentados se deduce que los morteros de escoria activada con $\mathrm{NaOH}$ son los más susceptibles al ataque por sulfatos y agua de mar. La leve reducción de resistencias observadas en estos morteros son debidas a la formación de productos de naturaleza expansiva, ya sean yeso y/o ettringita. Sin embargo, la cantidad de tales productos a los 180 días de inmersión en las disoluciones agresivas es baja si se compara con morteros de OPC, con contenidos en aluminatos normales.

En estos morteros no se ha identificado $\mathrm{Ca}(\mathrm{OH})_{2}$ (portlandita) libre y la proporción de iones $\mathrm{Ca}^{2+}$ presentes en la fase acuosa de los mismos es muy baja $(10-20 \mathrm{mg} / \mathrm{l})(28)$, aunque si se forman carbonatos cálcicos desde los primeros días de ensayo. Esto significa que hay poco calcio disponible $y$, por lo tanto, explica el bajo contenido de yeso formado, y detectado en los morteros de escoria activada con waterglass y $\mathrm{NaOH}$ conservados durante 90 días en agua de mar. La
From these results is inferred that $\mathrm{NaOH}$ activated slag mortars are more sensitive to sulfates and to sea water attack. The slight decrease in strength observed in those mortars conserved in aggressive solutions is due to the formation of products of expansive character, either gypsum and/or ettringite. Besides of this, the amount of such products at 180 immersion days into aggressive solutions is low compared to OPC mortars, with normal aluminates content.

In those mortars, no free $\mathrm{Ca}(\mathrm{OH})_{2}$ (portlandite) war identified, and the proportion of $\mathrm{Ca}^{2+}$ ions found in their aqueous phase is very low (10-20 mg/l) (28), although from early test-time calcium carbonate were already formed. This fact indicates that available calcium is scarce, which explains the low formation of gypsum detected in waterglass and in $\mathrm{NaOH}$ activated slag mortars inmersed during 90 days in sea water. The reaction of that gypsum with aluminates was 
formación de ettringita en los morteros de escoria activada con $\mathrm{NaOH}$ se puede justificar por la reacción de dicho yeso con aluminatos. En las pastas de escoria activada con $\mathrm{NaOH}$ se identifica un aluminato cristalino como la hidrotalcita $\left(\mathrm{Mg}_{6} \mathrm{Al}_{2} \mathrm{CO}_{3}(\mathrm{OH})_{16} \cdot 4 \mathrm{H}_{2} \mathrm{O}\right.$ y tampoco es descartable la presencia de aluminatos no cristalinos. Esa fase hidrotalcita no está presente en los morteros de escoria activados con waterglass. También este diferente comportamiento de los morteros de escoria activada con $\mathrm{NaOH}$ frente a los medios sulfáticos se puede explicar, en parte, por las diferencias estructurales y composicionales del silicato cálcico hidratado formado respecto al correspondiente en los morteros de escoria activada con waterglass. El primero tiene una mayor ordenación estructural, menor polimerización y mayor relación $\mathrm{C} / \mathrm{S}$ (es decir, es menos ácido) (21); todo ello explica su mayor susceptibilidad al ataque químico.

En los morteros de mezclas de escoria y ceniza volante activadas alcalinamente se identifica hidrotalcita como fase cristalina; sin embargo no se detectan productos de interacción con las disoluciones agresivas. Estos morteros tienen unos poros de tamaño muy pequeño dificultando el acceso de las disoluciones agresivas.

\subsection{Método ASTM C1012}

En las Tablas 7 y 8 se presentan los resultados de los ensayos realizados, de acuerdo con la especificación de la norma ASTM C1012 (26), en probetas de mortero de la escoria activada alcalinamente con waterglass de concentración $4 \%$ en $\mathrm{Na}_{2} \mathrm{O}$ en masa de escoria. El análisis de estas tablas indica que las probetas de mortero de escoria activada con waterglass no presentan un cambio apreciable en las dimensiones, obteniéndose valores máximos de sólo el ,.016\% de expansión a edad de 200 días de exposición a $\mathrm{Na}_{2} \mathrm{SO}_{4}$. Se puede notar que a pesar del buen comportamiento del mortero de cemento Portland las expansiones son menores en las mezclas de escoria siderúrgica activada alcalinamente y dado el bajo valor del porcentaje de expansión obtenido, esta expansión puede considerarse prácticamente insignificante. Douglas et al. (29) reportan resultados similares para especimenes de hormigón conservados en disolución de $\mathrm{Na}_{2} \mathrm{SO}_{4}$ al $5 \%$ durante 120 días.

Cabe anotar que este método, ASTM C1012, recomendado tanto para cementos Portland puros como adicionados, corresponde a una técnica de ensayo de agresividad de sulfatos de carácter acelerado, al exponer las probetas de mortero a una solución de concentración de $\mathrm{Na}_{2} \mathrm{SO}_{4}$ de $50 \mathrm{~g} / 1$ lo que equivale a 32.900 ppm de sulfato $\left(\mathrm{SO}_{4}{ }^{2-}\right)$ en agua, valor este que corresponde a una exposición muy severa (superior a 10.000 ppm) según los códigos (30). Estudios responsible for ettringite formation in slag mortars activated with $\mathrm{NaOH}$. In $\mathrm{NaOH}$ activated slag pastes an aluminum crystalline phase, hydrotalcite $\left.\left(\mathrm{Mg}_{6} \mathrm{Al}_{2} \mathrm{CO}_{3} \mathrm{OH}\right)_{16} \cdot 4 \mathrm{H}_{2} \mathrm{O}\right)$, was identified, but a possible presence of non-crystalline aluminates cannot be rejected. That hydrotalcite phase did not appear in slag mortars activated with waterglass. Also this different behavior of $\mathrm{NaOH}$ activated slag mortars in presence of sulphate environments is explainable, in part, by the structural and compositional differences of the hydrate calcium silicate formed as compared to the one obtained in waterglass activated slag mortars. The first has a major structural order, a lesser polymerization and a higher $C / S$ ratio (meaning that it is less acid) (21), all this explains its lesser resistance to sulfate attack.

In mortars made by alkaline activation of fly ash/slag mixture, hydrotalcite is identified as crystalline phase; however no reaction products with aggressive solutions were found. The high microporosity of these mortars can explain its behavior in sulfate environments.

\subsection{ASTM C1012 method}

Tables 7 and 8 show the results of tests carried out, according to the ASTM C1012 (26) standard specification, to mortar prisms prepared by alkaline activation of slag with waterglass $\left(4 \% \mathrm{Na}_{2} \mathrm{O}\right.$ in slag mass). Analysis of these tables point out that waterglass activated slag mortars did not undergo a remarkable dimensional change, resulting in expansion values of merely $0.016 \%$ after 200 days of exposure to $\mathrm{Na}_{2} \mathrm{SO}_{4}$ solution. It is noteworthy that in spite of the good behavior of Portland cement mortar, expansions were smaller in alkali-activated slag mortars. Douglas et al. (29) reported similar results for concrete specimens preserved in $\mathrm{Na}_{2} \mathrm{SO}_{4}$ solution at $5 \%$ during 120 days.

It has to be pointed out that ASTM C1012 method, recommended for pure Portland cements as much as for those with additions, corresponds to an accelerated test technique against sulfates aggressiveness that exposes mortar test samples to a solution of $50 \mathrm{~g} / \mathrm{l}$ $\mathrm{Na}_{2} \mathrm{SO}_{4}$ concentration, equal to $32.900 \mathrm{ppm}$ of sulfate $\left(\mathrm{SO}_{4}^{2-}\right)$ in water, which corresponds to a very severe exposure (higher than $10.000 \mathrm{ppm}$ ) according to regulations (30). Research on cement mortars had 
TABLA 7/TABLA 7

Porcentaje de expansión

(Percentage of expansion)

\begin{tabular}{|c|c|c|}
\hline $\begin{array}{c}\text { Tiempo de exposición en } \\
\mathrm{Na}_{2} \mathrm{SO}_{4} \text { (días) } \\
\text { Exposition time in } \\
\mathrm{Na}_{2} \mathrm{SO}_{4} \text { (days) }\end{array}$ & $\begin{array}{c}\text { Escoria Activada } \\
\text { Activated slag }\end{array}$ & $\begin{array}{c}\text { Cemento Portland } \\
\text { Porland cement }\end{array}$ \\
\hline 15 & 0 & 0 \\
\hline 45 & 0 & 0.011 \\
\hline 60 & 0 & 0.030 \\
\hline 90 & 0.010 & 0.033 \\
\hline 120 & 0.010 & 0.033 \\
\hline 140 & 0.016 & 0.033 \\
\hline 175 & 0.017 & 0.097 \\
\hline 200 & 0.016 & 0.110 \\
\hline
\end{tabular}

TABLA 8/TABLE 8

Resistencia a la compresión, Mpa

(Compressive strengths, Mpa)

\begin{tabular}{|c|c|c|c|c|}
\hline \multirow{2}{*}{$\begin{array}{c}\text { Tiempo de exposición } \\
\text { (días) }\end{array}$} & \multicolumn{2}{|c|}{$\begin{array}{c}\text { Escoria Activada } \\
\text { Activated slag }\end{array}$} & \multicolumn{2}{|c|}{$\begin{array}{c}\text { Cemento Pórtland } \\
\text { Porland cement }\end{array}$} \\
\cline { 2 - 5 } Exposition time (days) & $\begin{array}{c}\text { Agua } \\
\text { Water }\end{array}$ & $\mathrm{Na}_{2} \mathrm{SO}_{4}$ & $\begin{array}{c}\text { Agua } \\
\text { Water }\end{array}$ & $\mathrm{Na}_{2} \mathrm{SO}_{4}$ \\
\hline 45 & - & 40.5 & 39.3 & 34.9 \\
\hline 90 & - & 75.1 & 47.9 & 38.9 \\
\hline 120 & 100.7 & 85 & 51.8 & 42.5 \\
\hline 140 & 100.3 & 90.5 & 53.3 & 43.1 \\
\hline 200 & 83.0 & 92.5 & 54.9 & 44.5 \\
\hline
\end{tabular}

realizados en morteros de cemento por otros investigadores aplicando el mismo método ASTM C1012 recomiendan límites máximos de expansión a 6 meses de $0,05 \%$ para catalogar un material como de alta resistencia a sulfatos y $0.10 \%$ para moderada resistencia (31). Los porcentajes de expansión obtenidos en esta investigación indican que la escoria activada alcalinamente con waterglass corresponde a un material de alta Resistencia a los sulfatos.

En el comportamiento mecánico resistente se observa una leve disminución de la resistencia de las probetas expuestas al medio de sulfatos con respecto a las conservadas en agua. Sin embargo, este efecto para los morteros de escoria activada es apenas de un $10 \%$ a edades de 140 días, mientras que para los morteros del cemento Portland de referencia es del 19\%. A la edad de 200 días los resultados promedios de resistencia a la compresión de las probetas de escoria activada alcalinamente expuestos en los dos medios son comparables e incluso superiores para aquellas probetas been carried out by different researchers by applying the same ASTM C1012 method. They recommend a maximum expansion limit of $0.05 \%$ at 6 months for $a$ material to be classified as high resistance against sulphates, and $0.10 \%$ as medium strength (31). Expansion percentages obtained in this research indicate that waterglass activated slag mortars are a high sulfate resistant material.

Mechanical behavior bears a slight decrease of strength of samples in contact with sulfates environment if compared to those conserved in water. However, this effect when activated slag mortars were inmersed scarcely of $10 \%$ at 140 days, while in cement mortars is $19 \%$. At 200 days, average compressive strength of alkali-activated slag mortar specimens immersed in any of environments (sulfates and water), were higher than strength of Portland Cement prisms submitted to sulphates action. It is remarkable that no fissures provide evidence of the chemical resistance of 
sometidas a la acción de sulfatos. Cabe anotar, que sobre las probetas no se observan fisuras que indiquen un ataque del medio, por el contrario, el nivel de absorción se reduce en el tiempo, esto concuerda con lo observado en los ensayos de porosidad, en cuanto a un refinamiento de los poros que contribuyen a hacer menos permeable el material y, por tanto, más resistente al ataque de los sulfatos. De otra parte, los especimenes cúbicos de morteros de escoria activada alcalinamente expuestos a sulfatos al momento del ensayo de resistencia fracturan en la forma especifica de un material de muy alta resistencia.

\section{CONCLUSIONES}

La principal conclusión que se puede extracr del presente trabajo es la elevada estabilidad química frente al ataque por sulfatos y agua de mar de los morteros de escoria activada alcalinamente y de los morteros de mezcla de escoria y ceniza activados alcalinamente.

En los morteros de escoria activada con waterglass se ha comprobado a través de dos métodos diferentes la alta estabilidad frente a los sulfatos, por lo quel puede ser considerado como un material de alta resistencia a los sulfatos.

Los morteros de escoria activada con $\mathrm{NaOH}$ son los más susceptibles al ataque por sulfatos y agua de mar, con formación de yeso y ettringita en ambos medios. La formación de estos compuestos en muy baja proporción explica la leve disminución de las resistencias mecánicas.

Los morteros de mezclas de escoria y ceniza volante activadas alcalinamente evidencian una alta resistencia al ataque por sulfatos y agua de mar. Ello se explica por la elevada microporosidad de estos morteros.

\section{AGRADECIMIENTOS}

Los autores agradecen a la CICYT y al Ministerio de Ciencia y Tecnología la financiación de los proyectos MAT98-0792 y MAT2001-1490, sin cuyos fondos no hubiera sido posible realizar este trabajo. Los autores también agradecen a J. L. García y a A. Gil su colaboración en la realización de los ensayos mecánicos. the prisms; on the contrary, the absorption level decreased with time, which is in accordance with observation on porosity tests, since a pore refinement contributes to a less permeable material and therefore to the increment of the resistance to sulfates attack. On the other hand, cubical prisms of alkali-activated slag that underwent sulpfte exposure broke during the strength test in the same specific way as a very high strength material do.

\section{CONCLUSIONS}

The most important conclusion drawn from this work is that alkali-activated slag mortars and mortars prepared from an alkaline activation of a mixture of fly ash/slag have a high chemical resistance to sulfates and also to seawater attack.

Hardened waterglass activated slag mortars presented high stability against sulfates. This was proved through different methods. This material have to be registered as a high sulfate resistant material.

$\mathrm{NaOH}$ activated slag mortars were the most sensitive to sulphates and to sea water attack, forming gypsum and ettringite in both environments. The scarce formation of these compounds produced a low decrease of their mechanical strengths.

Alkaline activation of fly ash/slag mortars have a high resistance to sulfates and sea water attack. The pore refinement of these mortars explains this fact.

\section{ACKNOWLEDGEMENTS}

Authors thank the CICYT and MCyT for funding the projects MAT98-0792 and MAT2001-1490, which support made possible to complete this work. Authors also thank J. L. Garcia and A. Gil for their collaboration in the process of mechanical testing.

\section{REFERENCIAS}

(1) G.J. Osborne (1999). "Durability of portland blast-furnace slag cement concrete". Cem. Concr. Composites, 21, 11-21.

(2) V. D. Glukhovsky, G.S. Rostovskaja, G.V. Rumyna (1980) “High strength slag-alkaline cements”. $7^{\text {th }}$ Intern. Congr. Chem. Cem. (Paris), 3, V-164-168.

(3) V. D. Glukhovsky, Y. Zaitsev, V. Pakhonow. (1983) " Slag-alkaline cements and concretes structures, properties, technological and economical aspects of the use". Sil. Ind. 10, 197-200. 
(4) S. D. Wang, K. Scrivener. (1995) “Hydration products of alkali activated slag cement”. Cem. Concr. Res, Vol. 25, n 3, pp. 561571 .

(5) C. Shi, R. L. Day. (1995) “A calorimetry study of early hydration of alkali-slag cements”. Cem. Concr. Res, Vol. 25, n 6, pp. 13331346.

(6) A. Fernández-Jiménez, F. Puertas (1998) "Determination of kinetic equations of alkaline activation of blast furnace slag by means of calorimetric data". J. Therm. Anal. Vol. 52, pp. 945-955.

(7) Z. Huanhai, W. Xuequan, X. Zhongzi, T. Mingshu. (1993) “Kinetic study on hydration of alkali-activated slag”. Cem. Concr. Res., Vol. 23, $\mathrm{n}^{\circ} 6$, pp. 1253-1258.

(8) C. Shi (1996). "Strength, pore structure and permeability of alkali-activated slag mortars". Cem. Concr. Res. Vol. 26, n 12 , pp. 1789-1799.

(9) S.D. Wang, K. Scrivener, P.L. Pratt. (1994) "Factors affecting the strength of alkali-activated slag”. Cem. Concr. Res. Vol 24, ${ }^{\circ}$ 6, pp.. 1033-1043.

(10) B. Talling, J. Brandstetr. (1989). "Present state and future of alkali-activated slag concretes". $3^{\text {rd }}$ Intern. Conf. On Fly-ash, Silica Fume, Slag, and Natural Pozzolans in Concrete, Norway, Vol. 2, SP 114-74, pp. 1519-1546.

(11) J. Malopepszy, M. Petri, (1986) “High strength slag-alkaline binder”. $8^{\text {th }}$ Inter. Congr. Chem. Cem. Rio de Janeiro, Vol. 4, pp. 108-111.

(12) A. Fernández-Jiménez, J.G. Palomo, F. Puertas (1999) “Alkali-activated slag mortars. Mechanical strength behaviour”. Cem. Concr. Res, Vol. 29, pp. 1313-1321.

(13) P.V. Krivenko (1999) “Alkaline cements and concretes: problems of durability". $2^{\text {nd }}$ Intern. Conf. Alkaline Cements and Concretes (Kiev).

(14) J. Pera, M. Chabannet (1995) "Durability of alkali-activated slag cements". Procee. Mat. Res. Society. Symp. On "Mechanisms of Chemical Degradation of Cement-based System (Boston), pp. 281-288.

(15) J. Deja, J. Malolpeszy (1989) "Resistance of alkali-activated slag mortars to chloride solution". 3 rd Intern. Conf. On Fly-ash, Silica Fume, Slag, and Natural Pozzolans in Concrete, Norway, Vol. 2, SP 114-75, pp. 1547-1563.

(16) K. Byfors, G. Klingstedt, V. Lehtonen, H. Pyy, L. Romben, (1989) "Durability of concrete made with alkali activated slag". $3^{\text {rd }}$ Intern. Conf. On Fly-ash, Silica Fume, Slag, and Natural Pozzolans in Concrete, Norway, Vol 2, pp. 1429-1466.

(17) T. Bakharev, J..G. Sanjayan, Y.-B. Cheng (2001) "Resistance of alkali-activated slag concrete to alkali-aggregate reaction". Cem. Concr. Res, Vol. 31, pp. 331-334.

(18) P. V. Krivenko (1992) “Alkaline cements". $9^{\text {th }}$ Intern. Congr. Chem. Cem. (New Delhi), Vol4, pp 482-488.

(19) S. D. Wang, X.C. Pu, K.L. Scrivener, P.L. Pratt (1995) "Alkali-activated slag cement and concrete: a review of properties and problems". Adv. Cem. Res, Vol. 7, n² 27, pp. 93-102.

(20) F. Puertas (1995) "Cementos de escorias activadas alcalinamente: situación actual y perspectivas de futuro". Materiales de Construcción, Vol. 45, n²39, pp. 53-64.

(21) A. Fernández-Jiménez (2000) "Cementos de escorias activadas alcalinamente: influencia de las variables y modelización del proceso". Doctoral Thesis. Universidad Autónoma de Madrid.

(22) R. D. Hooton, J.J. Emery (1983) "Glass content determination and strength development preditions for vitrified blast furnace slag". First Int. Conf. Fly Ash, Silica Fume, Slag and other Mineral by products in concrete. Montebello, Quebec, Canada, SP 79-SO, pp. 943-962.

(23) British Standard Institution BSI BS*6699 92. Specification for Ground granulated blastfurnace slag for use with portland cement (1996).

(24) F. Puertas, S. Martínez-Ramírez, S. Alonso, T. Vázquez (2000) “Alkali-activated fly ash/slag cement. Strength behaviour and hydration products". Cem. Concr. Res, Vol. 30, pp. 1625-1632.

(25) A. Kock, H. Steinegger (1960) “Ein Schnellprüfverfahren für Zemente auf ihr verhalten bei sulfatangriff”. ZKG Int., 13 n $7, \mathrm{pp}$. 317-324.

26) ASTM C1012 (1989) “Test Method for Length Change of Hydraulic-Cement Mortars Exposed to a Sulfate Solution", Annual Book of ASTM Standards, Vol. 04.02, pp. 473-477.

27) S. Diamond (2000) "Mercury porosimetry. An inappropiate method for the measurement of pore size distributiens in cementbased materials". Cem. Concr. Res. Vol. 30, pp. 1517-i525.

28) J. C. Kim, S.Y. Hong (2001) "Liquid concentration changes during slag cement hydration by alkali activation". Cem. Concr. Res. Vol. 31, pp. 283-285.

29) E. Douglas, A.Bilodeau, V.M. Malhotra (1992) “Properties and Durability of alkali Activated Slag”. ACI Materials Journal, Vol. $89, \mathrm{n}^{\circ} 5$, pp. 509-516.

30) ACI 201.2R-92 (1997) "Guide to Durable Concrete", ACI Manual of Concrete Practice, Part 1. Materials and General Properties of Concrete.

31) T. Patzias (1991) “The development of ASTM C1012 with Recommended Acceptance Limits for Sulphate Resistance of Hydraulic Cements", Cement, Concrete and Aggregates, Vol. 13, $\mathrm{N}^{\circ} 1$. 